\title{
Pregnancy outcomes in adult patients with dermatomyositis and polymyositis
}

\author{
Kathleen D. Kolstad, MD, PhD ${ }^{a}$, David Fiorentino, MD, $\mathrm{PhD}^{\mathrm{b}}$, Shufeng $\mathbf{L i}, \mathrm{MS}^{\mathrm{b}}$, Eliza F. \\ Chakravarty, MD, MS $^{\mathrm{c}}$, and Lorinda Chung, MD, MS ${ }^{\mathrm{a},{ }^{,}}$ \\ aDivision of Immunology and Rheumatology, Department of Medicine, Stanford University School \\ of Medicine, Palo Alto, CA \\ ${ }^{b}$ Department of Dermatology, Stanford University School of Medicine, Redwood City, CA \\ ${ }^{\mathrm{C}}$ Arthritis and Clinical Immunology Research Program, Oklahoma Medical Research Foundation, \\ Oklahoma City, OK
}

\begin{abstract}
Objective-The idiopathic inflammatory myopathies dermatomyositis (DM) and polymyositis (PM) are autoimmune diseases that can affect females of childbearing potential. We assessed pregnancy outcomes in DM and PM patients compared with the general obstetric population.
\end{abstract}

\begin{abstract}
Methods-The Nationwide Inpatient Sample (NIS) (1993-2007) was used to identify deliveryassociated hospitalizations in women with DM or PM (DM/PM, $n=853)$. Controls were from the general obstetric population delivery-associated hospitalizations matched to each case by year of delivery. Pregnancy outcomes included hospital length of stay (LOS), hypertensive disorders (HTN), premature rupture of membranes (PROM), intrauterine growth restriction (IUGR), and cesarean delivery. Multivariate regression analyses were performed using maternal age, race/ ethnicity, and diabetes mellitus as covariates.
\end{abstract}

\begin{abstract}
Results-On multivariate analysis, patients with DM/PM had longer LOS compared to controls $(p<0.001)$. DM/PM was associated with an increased risk of hypertensive disorders compared to controls (OR $=2.90,95 \%$ CI: 2.00-4.22). There were no differences in rates of PROM, IUGR, or cesarean section in patients with DM/PM compared with controls. Independent of a DM/PM diagnosis, African-American race, older age, and diagnosis of diabetes increased the hospital LOS $(p<0.001)$. African-American race and diabetes increased the risk of hypertensive disorders (OR $=1.38,95 \%$ CI: $1.19-1.60 ; \mathrm{OR}=2.94,95 \%$ CI: $2.04-4.23$, respectively) compared to controls .
\end{abstract}

Conclusion-These data suggest that patients with inflammatory myopathies are at increased risk of hypertensive disorders of pregnancy and longer length of hospitalization. Vigilant monitoring of blood pressure is advisable in pregnant patients with DM or PM.

\footnotetext{
*Correspondence to: VA Palo Alto Health Care System, 3801 Miranda Avenue, Palo Alto, CA 94304, shauwei@ stanford.edu (L. Chung).

Appendix A. Supplementary material: Supplementary data are available in the online version of this article at http://dx.doi.org/ 10.1016/j.semarthrit.2017.11.005.
} 


\section{Keywords}

Dermatomyositis; Polymyositis; Pregnancy; Inflammatory myopathy; Hypertension; Nationwide inpatient sample

\section{Introduction}

Dermatomyositis (DM) and polymyositis (PM) are autoimmune inflammatory myopathies characterized by proximal symmetric muscle weakness and, in the case of DM, a variety of characteristic cutaneous manifestations. Women are twice as likely to be affected as men and the female to male ratio has been estimated to increase from 1.5-2:1 to 5:1 during childbearing years [1]. In addition, there have been case reports of disease onset during pregnancy or in the post-partum state, raising the question as to whether the disease can be triggered by pregnancy [2-4].

Inflammatory myopathies can be complicated by pulmonary, cardiovascular, and metabolic disease [5-8]. Pulmonary disease is the most common complication, primarily secondary to interstitial lung disease, but can also be related to pulmonary arterial hypertension, aspiration pneumonitis, or drug-induced pneumonitis [5]. Regarding cardiovascular complications, patients can develop arrhythmias, coronary artery disease, or congestive heart failure $[7,8]$. There is also evidence that patients with inflammatory myopathies are at greater risk of HTN and diabetes [6]. Such underlying co-morbidities have the potential to negatively impact pregnancy outcomes.

High disease activity and associated co-morbidities could potentially increase the risk of pregnancy complications in women with DM or PM. Little is known about pregnancy outcomes in patients with inflammatory myopathies. Small studies and case reports suggest that flares of disease activity during pregnancy may result in poor fetal outcomes such as intrauterine growth restriction (IUGR), prematurity, or fetal loss [4,9-12]. There are limited studies evaluating pregnancy outcomes in patients with DM/PM at the population level [13]. The goal of this study was to utilize a US-based nationwide dataset to assess whether women with DM/PM are at greater risk of pregnancy complications compared to women in the general obstetric population.

\section{Materials and Methods}

\section{Patient Cohort and Outcomes}

Inpatient hospitalizations from 1993 to 2007 were analyzed using the Nationwide Inpatient Sample (NIS) Dataset to assess obstetric outcomes in women with DM and PM [14]. The NIS is the largest publicly available all-payer health care database containing information on inpatient hospitalizations in the United States. The NIS extracts hospital data to approximate a 20\% sample of all US community hospitals. Data extracted by the NIS includes discharge abstracts containing information on primary and secondary diagnoses and procedures, patient demographics, hospital characteristics, payment source, discharge status, length of stay, and severity and comorbidity measures $[14,15]$. An institutional review board waiver 
was obtained, as the data used are publicly available and patient identifiers are not included in the dataset.

We used 15 years of NIS data (1993-2007) to estimate total delivery-associated obstetric hospitalizations for patients with the inflammatory myopathies DM $(n=454)$ and PM $(n=$ 399). This time frame was used to obtain sufficient data, as DM and PM are rare diseases. The control group included obstetric delivery-associated hospitalizations without DM or PM. Totally, 100 randomly selected delivery-associated hospitalizations from the general obstetric population were matched to each DM/PM delivery-associated hospitalization by year of delivery $(n=101,123)$. International Classification of Diseases, Ninth Revision, Clinical Modification (ICD-9CM) codes in discharge summaries were used to identify patient groups (Supplementary Table 1). The groups included DM, PM, and the control group. Cases and controls with other connective tissue disease diagnoses including systemic lupus erythematosus (SLE), systemic sclerosis, and rheumatoid arthritis were excluded.

Discharge summaries from each delivery-associated hospitalization were analyzed to identify maternal age, race/ethnicity, hospital length of stay (LOS), principal and secondary diagnoses, and procedures. The diagnoses and procedures identified by ICD-9 code for this study include pre-gestational and gestational diabetes mellitus, hypertensive disorders of pregnancy (HTN), premature rupture of membranes (PROM), intrauterine growth restriction (IUGR), and cesarean delivery. Hypertensive disorders of pregnancy include gestational hypertension, pre-existing hypertension, pre-eclampsia, and eclampsia (Supplementary Table 1).

\section{Statistical Analyses}

We compared maternal age, race/ethnicity, and presence of a diagnosis of diabetes mellitus between the DM/PM group and the control group. We randomly selected 100 controls for each case from the general obstetric population matching by the year of delivery. Differences in means were compared with Student's $t$-test and differences in proportions with Rao-Scott chi-square test. Univariate analyses were employed to compare LOS and adverse pregnancy outcomes (HTN, PROM, IUGR, and cesarean delivery) between the DM/PM group and controls. Multivariate analyses were also performed to estimate the effect of a diagnosis of $\mathrm{DM} / \mathrm{PM}$ on LOS (linear regression) and on the likelihood of diagnosis of the above mentioned adverse pregnancy outcomes (logistic regression). Additional co-variates for multivariate regression models were maternal age, race/ethnicity, and diabetes mellitus. The above analyses were performed on the subset of hospitalizations that resulted in delivery. We did not include an analysis of outcomes associated with antenatal hospitalizations, as we were unable to correct for multiple hospitalizations of individual patients. All analyses were weighted to account for the survey sampling weight to produce national estimates. Analyses were performed using SAS (version 9.4, SAS Institute, Inc., Cary, NC). 


\section{Results}

\section{Population estimates and demographics}

Table 1 shows population estimates of delivery-associated hospitalizations and cesarean deliveries of controls and women with DM and PM. We compared the outcomes and complications associated with 101,123 deliveries in the general obstetric population to 853 occurring in women with DM/PM.

African-Americans made up a higher percentage of patients with inflammatory myopathies, while Caucasians were more frequent in the control group $(p<0.001)$. Patients in the $\mathrm{DM} / \mathrm{PM}$ group were more likely to have diabetes and were older compared to controls ( $p<$ $0.001, p<0.001)$ (Table 2).

\section{Obstetric outcomes}

Obstetric outcomes of DM/PM patients during delivery-associated hospitalizations were compared to controls by univariate analysis. Patients with DM/PM were more likely to have a longer LOS (mean of $4.3 \pm 0.07$ days compared to $2.5 \pm 0.01$ days in controls; $p<0.001$ ) and hypertensive disorders (20.9\% of DM/PM patients compared 7.4\% of controls; $p<$ $0.001)$. DM/PM patients trended toward an increased risk of IUGR ( $3.1 \%$ compared to $1.4 \% ; p=0.0500$ ). PROM and cesarean deliveries were not significantly different in DM/PM patients compared to controls in this analysis (Table 3).

Multivariate regression analyses were performed as shown in Table 4. After adjustment for covariates, a diagnosis of DM/PM was associated with an increased risk of HTN $(\mathrm{OR}=$ 2.90, 95\% CI: 2.00-4.22). A sensitivity analysis excluding pre-existing hypertension confirmed the increased risk of hypertensive disorders of pregnancy in DM/PM compared with the general population ( $\mathrm{OR}=2.18,95 \% \mathrm{CI}: 1.37-3.46)$. There were no significant differences in rates of PROM, IUGR, or cesarean delivery in DM/PM patients compared with controls. Linear regression analysis also showed that patients with DM/PM have longer hospital LOS ( $\beta=1.73,95 \%$ CI: 1.55-1.92) than controls, even after controlling for increased risk of hypertensive disorders $(\beta=1.54,95 \%$ CI: 1.44-1.65). Risk of hypertensive disorders did not differ when comparing DM to PM patients (including pre-existing hypertension $\mathrm{OR}=0.71,95 \% \mathrm{CI}$ : $0.35-1.44$; excluding pre-existing hypertension $\mathrm{OR}=$ 0.85, 95\% CI: $0.32-2.26)$. There were also no differences in PROM or cesarean delivery when comparing DM to PM patients, however, there was a difference in hospital LOS ( $\beta=$ $-0.87,95 \%$ CI: -1.07 to -0.68 ) and IUGR (OR $=0.33,95 \%$ CI: $0.12-0.95$ ). Diabetes, increasing age, and African-American race were also associated with increased LOS. Diabetes and African-American race increased the risk of hypertensive disorders. There was an increased risk of cesarean delivery in the other races group ( $\mathrm{OR}=1.10,95 \% \mathrm{CI}$ : 1.004 $1.20)$, with increasing age $(\mathrm{OR}=1.044,95 \% \mathrm{CI}: 1.038-1.05)$, and with diabetes $(\mathrm{OR}=1.49$, 95\% CI: 1.11-1.99). Finally, there was a decreased risk of PROM in the other races group $(\mathrm{OR}=0.83,95 \%$ CI: 0.69-0.99) $($ Table 4$)$.

We performed additional sensitivity analyses. First, when matching controls and DM/PM cases by year of delivery, age at delivery, and diabetes status, our results on univariate and multi-variate analyses remained unchanged. We also assessed for the effects of time period 
on our analyses given that trends in obstetric and rheumatologic care have changed over time. We assessed outcomes in patients treated during the time periods from 1993 to 1999 and 2000 to 2007. We estimated 399 and 453 deliveries in these periods, respectively. On univariate analysis there was no difference in findings when compared to our original results. DM/PM patients had significantly longer hospital LOS and a higher percentage of patients with hypertensive disorders of pregnancy for both time periods $(p<0.001)$. A trend of increasing rates of cesarean section was noted in the more recent time period for both control and DM/PM patients. On multivariate analysis DM/PM patients continued to be at greater risk of longer hospital LOS and hypertensive disorders of pregnancy in both time periods. This finding is similar to our primary results, however, it was noted that the hospital LOS decreased and the HR for hypertensive disorders of pregnancy increased during the 2000-2007 time period (Table 5).

\section{Discussion}

Women with rheumatologic diseases often have increased risks of pregnancy complications, including preeclampsia, preterm birth, cesarean section, and IUGR [16,17]. However, it has been unclear whether these findings extend to women with inflammatory myopathies given the rarity of these diseases. DM and PM can affect women of childbearing age, however, studies regarding their impact on pregnancy have been limited by sample size and, in many cases, restricted to academic referral centers. In addition, the majority of studies evaluating obstetric outcomes in women with inflammatory myopathies have focused on fetal outcomes with some conflicting results. Multiple case reports and small retrospective studies suggest that fetal outcomes are worse in active disease, with increased risk of spontaneous abortion and prematurity [9-13].

Few studies have focused on maternal and delivery associated complications among women with inflammatory myopathies. One retrospective cohort study of 51 patients found there was no difference in maternal or fetal complications comparing pregnancies before and after disease onset; however, this study was limited by size and the comparison was not made with the general population [18]. Another recent population-based study showed an increase in hypertensive disorders of pregnancy, antepartum hemorrhage, and cesarean deliveries in patients with inflammatory myopathies, however this study only included $17 \mathrm{DM} / \mathrm{PM}$ pregnancies [13]. Our goal was to overcome these limitations by using a large publicly available US database to estimate adverse pregnancy outcomes in women with DM/PM compared with the general obstetric population. Our findings suggest that patients with $\mathrm{DM} / \mathrm{PM}$ are at increased risk of certain obstetric complications compared to the general population.

We found that DM/PM patients are hospitalized longer during delivery and are at increased risk of hypertensive disorders, including preeclampsia and eclampsia, a finding that is consistent with prior smaller studies $[9,13]$. This result persisted after we excluded a diagnosis of pre-existing hypertension. This is an important point as hypertension is more common in non-pregnant patients with DM/PM compared to the general population [6]. In multivariate analyses, we did not find an increased risk of PROM, IUGR, or cesarean 
delivery in patients with DM/PM, outcomes that have not been extensively evaluated in this population previously.

Abnormal placentation and placental function have been implicated in the pathogenesis of hypertensive disorders of pregnancy and other complications of pregnancy [19,20]. If poor placentation is more common in women with inflammatory myopathies, a higher prevalence of IUGR, prematurity, and fetal loss may also be expected. Of those outcomes, our study only evaluated IUGR and DM/PM patients trended toward an increased risk of IUGR in univariate analysis, though this was not significant when correcting for potential confounding factors. Prior studies have suggested that patients with myositis are at greater risk of fetal loss and prematurity [5-9], thus, further studies would be helpful to elucidate whether pregnant women with DM/PM are at risk of abnormal angiogenesis and placental development and subsequent related fetal complications.

There are limitations of this study due to the rarity of inflammatory myopathies in the general population and the data set used. In order to overcome the limitation of the total number of patients with inflammatory myopathies available for evaluation, we sampled data over 15 years and combined DM and PM patients in the analyses. Data from control pregnancies were matched to the DM/PM cases by year of delivery to account for practice changes over time. Additionally, in a sensitivity analysis, our results remained the same when assessing outcomes by time period (1993-1999 and 2000-2007). There is no way to validate the diagnoses captured in this study because the data are de-identified. Our group previously found that ICD-9 codes 710.3 and 710.4 used in discharge summaries are $\$ 80 \%$ accurate in identifying cases of DM and PM, respectively (Narang et al. [21] International Classification of Diseases-Clinical Modification-9 Codes for the Diagnosis of Dermatomyositis and Polymyositis in Discharge Summaries: Evidence of Acceptable Validity. [abstract]. Arthritis Rheum 2011;63 Suppl 10:244). Furthermore, another recent study estimated a positive predictive value of $95 \%$ for the inpatient ICD-9 code 710.3 for the diagnosis of DM [22]. In order to avoid misclassification, we excluded patients with ICD-9 codes for other connective tissue diseases in addition to DM or PM. Therefore, we did not assess the influence of concomitant autoimmune disorders, such as SLE or systemic sclerosis, in our population that may influence obstetric outcomes [17].

The NIS dataset is primarily used for billing purposes and is therefore subject to coding errors and missing data. Indeed, race/ethnicity data were missing for $28 \%$ of hospitalizations. The dataset is de-identified and therefore information on disease activity during pregnancy, treatments received, autoantibody status, prior pregnancy complications, time of diagnosis in relation to pregnancy outcomes, and linkage of maternal-neonatal outcomes is not available. Spontaneous abortions also could not be assessed as the obstetric cases in our study were only identified after 20 weeks gestation and the majority of such losses occur earlier in pregnancy.

\section{Conclusion}

Despite the described limitations of the dataset, the major strength of this study is the number of pregnancies assessed, as inflammatory myopathies are rare diseases. We found 
that women with DM/PM are at greater risk of hypertensive disorders of pregnancy. Based on our results, pre-conception counseling, multi-disciplinary care with high-risk obstetricians, and blood pressure control is advisable in women with inflammatory myopathies who are pregnant or are planning to become pregnant.

\section{Supplementary Material}

Refer to Web version on PubMed Central for supplementary material.

\section{References}

1. Dourmishev, LA., Dourmishev, AL. Dermatomyositis: Advances in Recognition, Understanding and Management. Vol. 1. Berlin, Heidelberg: Springer Link; 2009. p. 29-36.Online Service

2. Harris A, Webley M, Usherwood M, Burge S. Dermatomyositis presenting in pregnancy. Br J Dermatol. 1995; 133:783-5. [PubMed: 8555034]

3. Kanoh H, Izumi T, Seishima M, Nojiri M, Ichiki Y, Kitajima Y. A case of dermatomyositis that developed after delivery: the involvement of pregnancy in the induction of dermatomyositis. $\mathrm{Br} \mathbf{J}$ Dermatol. 1999; 141:897-900. [PubMed: 10583175]

4. Rosenzweig BA, Rotmensch S, Binette SP, Phillippe M. Primary idiopathic polymyositis and dermatomyositis complicating pregnancy: diagnosis and management. Obstet Gynecol Surv. 2017; 44:162-70. in preparation.

5. Fathi M, Lundberg IE, Tornling G. Pulmonary complications of polymyositis and dermatomyositis. Semin Respir Crit Care Med. 2017; 28:451-8. in preparation.

6. Limaye VS, Lester S, Blumbergs P, Roberts-Thomson PJ. Idiopathic inflammatory myositis is associated with a high incidence of hypertension and diabetes mellitus. Int J Rheum Dis. 2010; 13:132-7. [PubMed: 20536597]

7. Lundberg IE. The heart in dermatomyositis and polymyositis. Rheumatology (Oxford, England). 2017; 45:18-21. in preparation.

8. Linos E, Fiorentino D, Lingala B, Krishnan E, Chung L. Atherosclerotic cardiovascular disease and dermatomyositis: an analysis of the nationwide inpatient sample survey. Arthritis Res Ther. 2013; 15:R7. [PubMed: 23298514]

9. Chopra S, Suri V, Bagga R, Thami MR, Sharma A, Bambery P. Autoimmune inflammatory myopathy in pregnancy. Medscape J Med. 2008; 10:17. [PubMed: 18324327]

10. Nagy-Vincze M, Vencovsky J, Lundberg IE, Dankó K. Pregnancy outcome in idiopathic inflammatory myopathy patients in a multicenter study. J Rheumatol. 2014; 41:2492-4.

11. Silva CA, Sultan SM, Isenberg DA. Pregnancy outcome in adult-onset idiopathic inflammatory myopathy. Rheumatology (Oxford, England). 2003; 42:1168-72.

12. Váncsa A, Ponyi A, Constantin T, Zeher M, Dankó K. Pregnancy outcome in idiopathic inflammatory myopathy. Rheumatol Int. 2007; 27:435-9. [PubMed: 17033833]

13. Chen JS, Roberts CL, Simpson JM, March LM. Pregnancy outcomes in women with rare autoimmune diseases. Arthritis Rheumatol. 2015; 67(12):3314-23. [PubMed: 26434922]

14. Healthcare cost and utilization project: overview of the Nationwide Inpatient Sample (NIS). URL: http://www.hcup-us.ahrq.gov/nisoverview.jsp

15. Steiner C, Elixhauser A, Schnaier J. The healthcare cost and utilization project: an overview. Eff Clin Pract. 2002; 5:143-51. [PubMed: 12088294]

16. Skomsvoll JF, Ostensen M, Irgens LM, Baste V. Pregnancy complications and delivery practice in women with connective tissue disease and inflammatory rheumatic disease in Norway. Acta Obstet Gynecol Scand. 2000; 79:490-5. [PubMed: 10857874]

17. Østensen M, Andreoli L, Brucato A, Cetin I, Chambers C, Clowse MEB, et al. State of the art: reproduction and pregnancy in rheumatic diseases. Auto-immun Rev. 2015; 14:376-86.

18. Pinal-Fernandez I, Selva-O'Callaghan A, Fernandez-Codina A, Martinez-Gomez X, RodrigoPendas J, Perez-Lopez J, et al. Pregnancy in adult-onset idiopathic inflammatory myopathy: report 
from a cohort of myositis patients from a single center. Semin Arthritis Rheum. 2014; 44:234-40. [PubMed: 24906908]

19. Furuya M, Ishida J, Aoki I, Fukamizu A. Pathophysiology of placentation abnormalities in pregnancy-induced hypertension. Vasc Health Risk Manag. 2008; 4(6):1301-13. [PubMed: 19337544]

20. Kroener L, Wang ET, Pisarsk MD. Predisposing factors to abnormal first trimester placentation and the impact on fetal outcomes. Semin Reprod Med. 2016; 34(1):27-35. [PubMed: 26696276]

21. Narang N, Fiorentino D, Krishnan E, Chung L. International classification of diseases-clinical modification-9 codes for the diagnosis of dermatomyositis and polymyositis in discharge summaries: evidence of acceptable validity. Arthritis Rheum. 2011; 63(Suppl 10):244. abstract.

22. Kwa MC, Ardalan K, Laumann AE, Nardone B, West DP, Silverberg JI. Validation of international classification of diseases codes for the epidemiologic study of dermatomyositis. Arthritis Care Res (Hoboken). 2017; 69(5):753-7. [PubMed: 27564726] 
Table 1

Population estimates for delivery associated hospitalizations by diagnosis

\begin{tabular}{lrcrc}
\hline Group & Deliveries & 95\% CI & Cesarean delivery & 95\% CI \\
\hline Control & 101,123 & $96,721-105,524$ & 28,784 & $27,345-30,222$ \\
DM & 454 & $356-551$ & 170 & $113-227$ \\
PM & 399 & $303-495$ & 165 & $106-223$ \\
DM + PM & 853 & $709-997$ & 335 & $253-417$ \\
\hline
\end{tabular}


Table 2

Demographics and comorbid conditions for delivery-associated hospitalizations in DM/PM patients compared to controls

\begin{tabular}{lrr}
\hline Variable & DM + PM & Control \\
\hline AGE & $28.8 \pm 0.19^{*}$ & $27.7 \pm 0.03$ \\
White (\%) & 42.1 & $57.2^{*}$ \\
African-American (\%) & $37.9^{*}$ & 15.1 \\
Other race (\%) & 20.0 & 27.7 \\
Diabetes (\%) & $4.1 *$ & 0.9 \\
\hline
\end{tabular}

* $p<0.001$ compared to controls. 

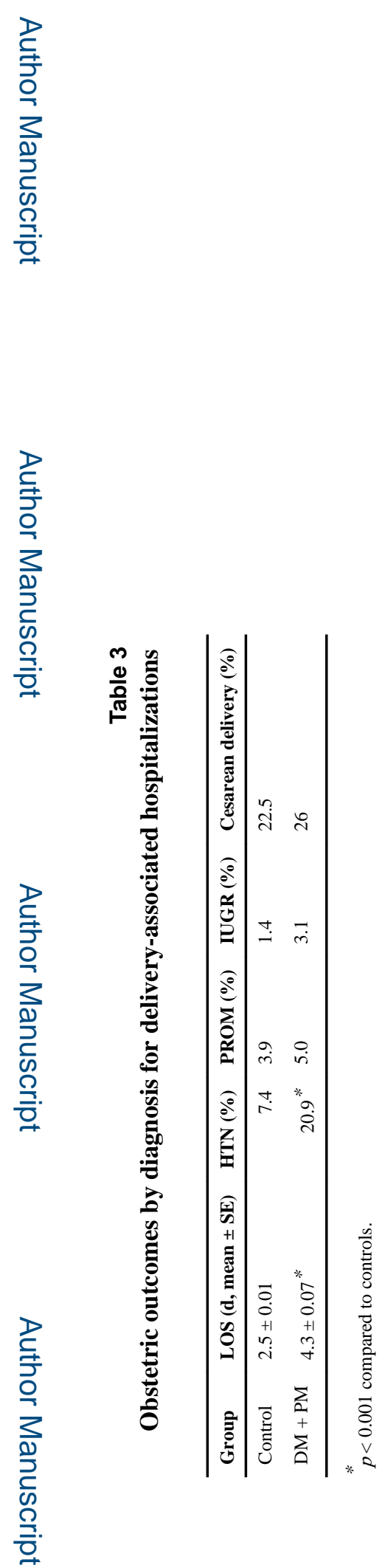

Semin Arthritis Rheum. Author manuscript; available in PMC 2018 June 01. 


\section{롤}

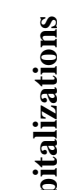

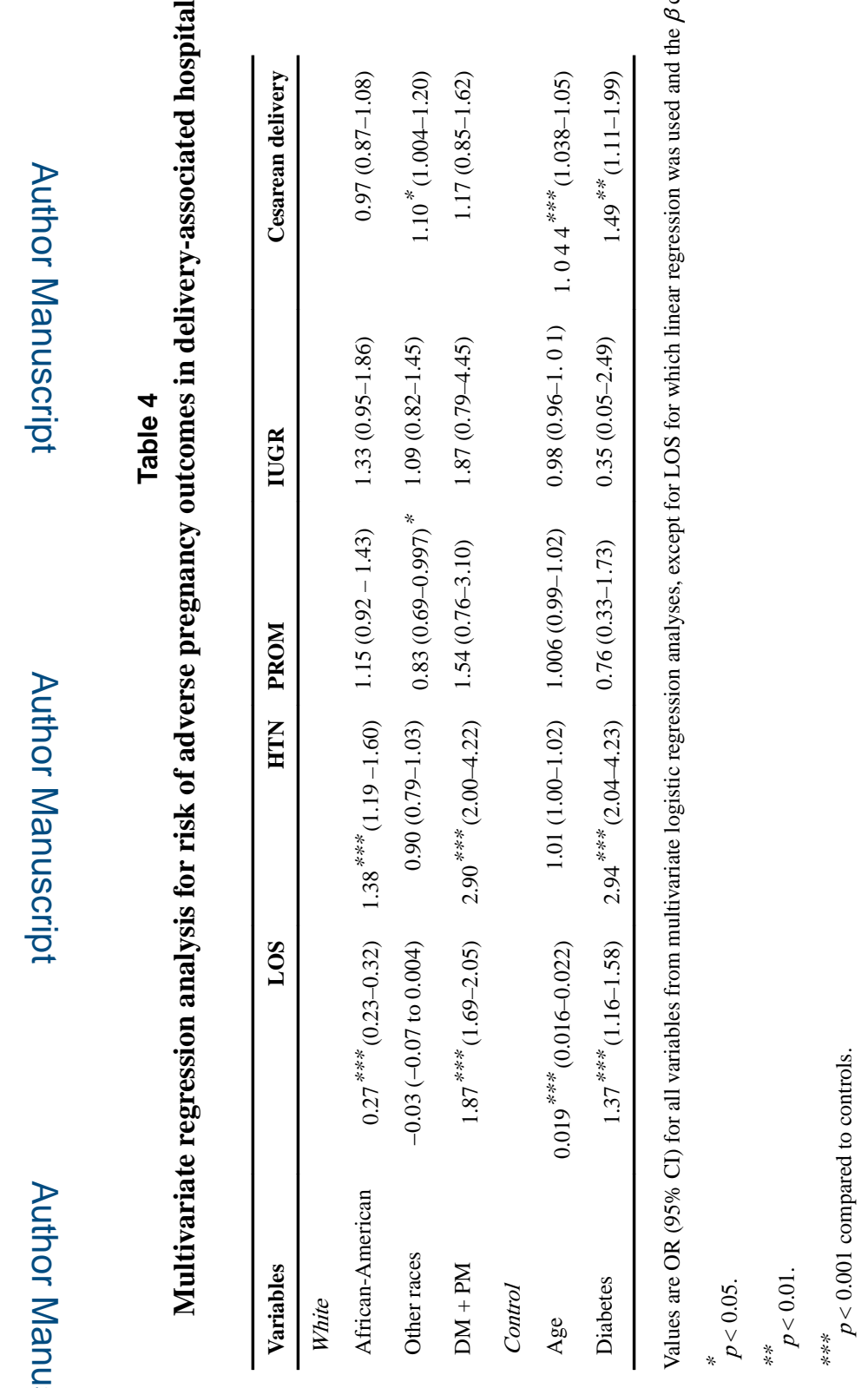

Semin Arthritis Rheum. Author manuscript; available in PMC 2018 June 01. 


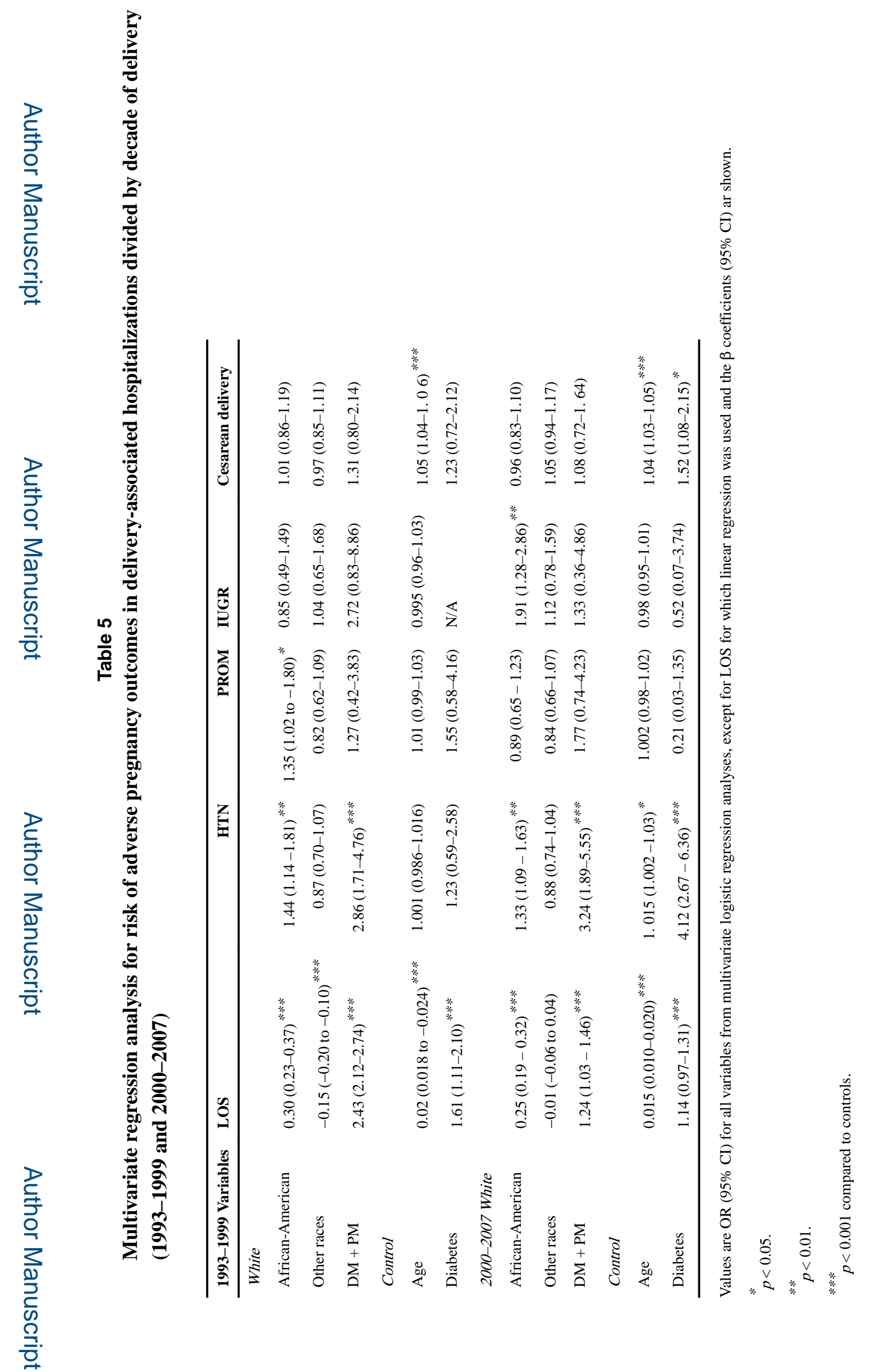

Semin Arthritis Rheum. Author manuscript; available in PMC 2018 June 01. 